\title{
Low Frequency Dielectric Study of PAPA-PVA-GR Nanocomposites
}

\author{
I. Latif, Taghreed B. Alwan, Ammar H. Al-Dujaili" \\ Department of Chemistry, College of Education, Ibn Al-Haytham, University of Baghdad, Baghdad, Iraq
}

\begin{abstract}
A new polymer poly[2, 2-bis ((4-acetylbenzoyloxy) methyl) propane-1,3-diyl bis(4-acetylbenzoate)] (PAPA) was synthesized by solution polymerization of pentaerythretole with terephthaloyl chloride. This polymer was characterized by FT-IR spectroscopy. Graphene (GR) was prepared using hydrothermal method. Evaluation of graphene structure was performed using FT-IR and XRD. Polymer composites were prepared by ultrasonic mixing of PAPA and PVA with different GR percentages. Composites films were prepared by a conventional solvent casting technique. The morphological image of the composite materials was studied by scanning electron microscopy (SEM) and the surface topography of these composite materials was studied by Atomic Force Microscopy (AFM). Effects of GR nanoparticles percentage on dielectric properties of PAPA/PVA/GR nanocomposites film at low frequency $(100 \mathrm{~Hz}-100 \mathrm{kHz})$ were studied.
\end{abstract}

Keywords Nanocomposite, Graphene, Polymer, Dielectric Properties

\section{Introduction}

Since the 2010 Nobel Prize in physics was awarded to Professors Andre Geim and Konstantin Novoselov[1,2] for their ground-breaking experiments on graphene and the attention of the scientist are growing daily to the single layer of graphite (GR) extraordinary thermal, mechanical, electrical and other properties. GR is a monolayer of $\mathrm{sp}^{2}-$ hybridized carbon atoms arranged in a two-dimensional lattice[3]. One of the most promising applications of this material is in polymer nanocomposites, chemically modified GR have been used to make functional polymer composites[4-7]. Chemical functionalization of GR surface by either oxidation procedure or physical adsorption/grafting protocols have been found to be a feasible and effective means for improving the dispersion of GR in organic and/or aqueous media. In addition, the attached functional groups may enhance the interfacial interactions between the GR and the polymer matrix. The advantage of modifying the GR surface by physical adsorption is that the structural integrity of the conjugated network remains unaltered, whereas formation of defects is observed after treatment of the graphitic nanostructures by oxidative conditions and/or grafting reactions.

In recent years, elastomeric materials with high dielectric constant have been considered for different functional applications such as artificial muscles, high charge-storage

* Corresponding author:

ahdujaili@yahoo.com (Ammar H. Al-Dujaili)

Published online at http://journal.sapub.org/nn

Copyright (C) 2012 Scientific \& Academic Publishing. All Rights Reserved capacitors and high- $\mathrm{K}$ gate dielectric for flexible electronics[2, 8]. Several methods have been explored in order to increase their dielectric permittivity although the most common approach involves the addition of high dielectric constant ceramics to the elastomeric matrix. This strategy usually requires high loading fractions and, hence, produces an unwanted increase of the system rigidity for the applications already mentioned[9-11]. In some other cases, dielectric constant increments have been met with relatively high loss tangent values $(\tan (\delta))$ and frequency dependence which is also undesirable for capacitor applications[12, 13]. Obtaining composites with both high dielectric permittivity and low loss tangent values at the same time is specially challenging due the interfacial polarization or Maxwell Wagner-Sillars (MWS) process. This mechanism occurs at the interface between materials with different permittivity and/or conductivities and involves rather high $\varepsilon^{\prime}$ and $\tan (\delta)$ values at low frequencies due to the accumulation of virtual charges at the filler/polymer interface[14]. Altering the interfacial interaction between filler and polymer matrix can regulate the dielectric contrast between matrix and filler and thus, prevent the MWS polarization[15-17]. Therefore, chemical modification of filler particles has to be taken into account in order to achieve high permittivity composites with low dielectric losses. Nevertheless, filler surface modifications can significantly raise the production costs and thus, make them unfeasible to be produced on large scale. Thermally expanded GR sheets are of great interest to overcome the aforementioned problems. The thermal reduction of the graphite oxide has the advantage to produce chemically modified graphene sheets (or so-called functionalized graphene sheets FGS) without the need of 
further modification steps. Besides, the huge aspect ratio of these carbon-based nanoparticles (experimental value 1850 $\mathrm{m}^{2} / \mathrm{g}$ )[18] reduces considerably the percolation threshold compared to any other type of high dielectric constant filler. Accordingly, very small loading fractions can offer interesting permittivity enhancements without adversely affecting the dielectric losses and mechanical properties of a given polymer matrix[19] show that FGS are an ideal candidate as high dielectric constant fillers in capacitor applications. The presence of remaining functional groups at their surface is able to improve the filler matrix compatibility, enhance the nanoparticle distribution and make them suitable to develop novel, flexible and easy to process capacitors with relatively high dielectric constant and low tan $(\delta)$ values[19].

To the best of our knowledge, there is no report on fabricating graphene/polymer composites by using noncovalently modified pristine graphene nanoplatelets. In addition, dielectric data such as dielectric permittivity, ac conductivity, and electric modulus have not been widely studied in graphene/polymer composites[15]. Accordingly, we prepared PAPA/PVA/GR nanocomposites via ultrasonic mixining and investigated their electrical behaviour at various low frequencies in details.

\section{Experimental}

\subsection{Materials}

All chemicals including sulfuric acid (98\%), hydrochloric acid (36 wt $\%$ ), hydrogen peroxide (30 wt $\%$ ), potassium permanganate, sodium nitrate were purchased from Merck Germany and pentaerythritol, terephthaloyl chloride, sodium hydroxide, PVA $\left(\mathrm{M}_{\mathrm{n}}=72000\right)$, and N,N-dimethylformamide (DMF) were purchased from Sigmae-Aldrich Chemicals and used as received. Graphene was prepared from natural flake graphite powder (Sinopharm Chemical Reagent Co., Ltd).

\subsection{Instruments}

For the characterization of GR, polymer and PAPA / PVA/GR nanocomposites the following instruments were used: Elemental analyzer $(\mathrm{C}, \mathrm{H}, \mathrm{N})$ (Perkin-Elmer model 2400). FT-IR spectrophotometer (8300 FT-IR Shimadzu Spectrophotometer), XRD (Shimadzu XRD-6000) with copper radiation $\left(\mathrm{Cu} \mathrm{K}_{\alpha}, 1.5406 \AA\right)$, Atomic Force Microscopy (AFM, AA 3000 Scanning probe Microscope), Scanning electron microscopy (SEM) (VEGA TE SCAN USA). For the dielectric properties was used Precision LCR meter HP 4274 A connected with HP 4275 A and Test Fixture HP $16047 \mathrm{~A}$ at frequency range $100 \mathrm{~Hz}$ to $100 \mathrm{kHz}$.

\subsection{Synthesis of Graphene Oxide (GO)}

Hummer method was used[20] to oxidize the graphite for the synthesis of graphene oxide (GO). First, $2 \mathrm{~g}$ graphite, $1 \mathrm{~g}$ sodium nitrate and $46 \mathrm{~mL}$ of sulfuric acid were mixed and strongly stirred at $0^{\circ} \mathrm{C}$ for $15 \mathrm{~min}$ in a $500 \mathrm{~mL}$ reaction flask immersed in ice bath. Then $6 \mathrm{~g}$ potassium permanganate was added slowly to the above solution and cooled for $15 \mathrm{~min}$. After this, the suspended solution was stirred continuously for $1 \mathrm{~h}$, and $92 \mathrm{~mL}$ of water was added slowly to the suspension for $10 \mathrm{~min}$. Subsequently, the suspension was diluted by $280 \mathrm{~mL}$ of warm water and treated with $10 \mathrm{~mL}$ of $\mathrm{H}_{2} \mathrm{O}_{2}(30 \%)$ to reduce residual permanganate to soluble manganese ions. Finally, the resulting suspension was filtered, washed with distilled water, and dried in a vacuum oven at $60^{\circ} \mathrm{C}$ for $24 \mathrm{~h}$ to obtain GO, Figure 1 .

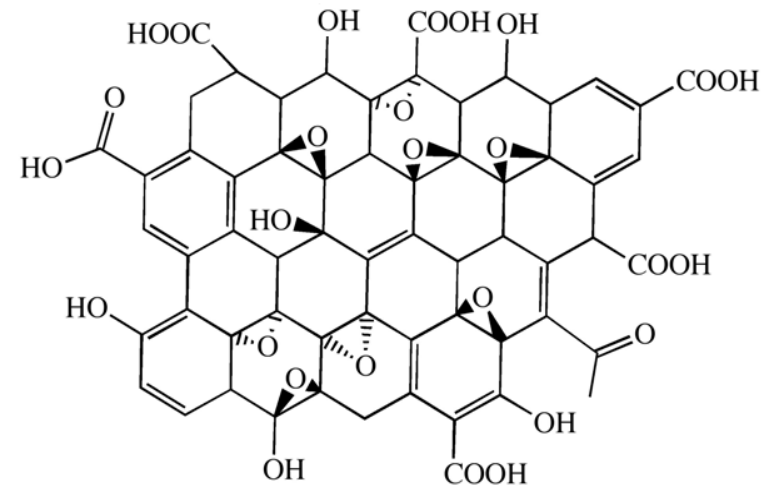

Figure 1. The proposed structure for GO

\subsection{Synthesis of Graphene (GR)}

Graphene was synthesized in a typical procedure, GO $(100 \mathrm{mg})$ was loaded in a $250 \mathrm{~mL}$ round bottomed flask and water $(100 \mathrm{~mL})$ was then added, yielding an inhomogeneous yellow-brown dispersion. This dispersion was sonicated using ( Soniprep 150 Plus) until it became clear with no visible particulate matter. Hydrazine hydrate $(1.00 \mathrm{~mL}, 32.1$ $\mathrm{mmol}$ ) was then added and the solution heated in an oil bath at $100^{\circ} \mathrm{C}$ under a water-cooled condenser for $24 \mathrm{~h}$ over which the reduced GO gradually precipitated out as a black solid. This product was isolated by filtration, washed continuously with water $(5 \times 100 \mathrm{~mL})$ and methanol $(5 \times 100 \mathrm{~mL})$, and dried in oven $\left(50^{\circ} \mathrm{C}\right)[21]$.

\subsection{Synthesis of Poly[2,2-bis((4-acetylbenzoyloxy) methyl) Propane-1,3-diyl bis(4-acetylbenzoate)] (PAPA)}

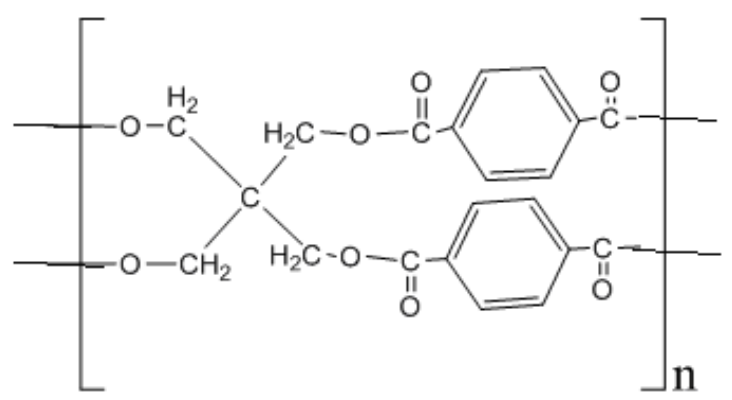

(PAPA)

To a stirring solution of pentaerythritol $(1.36 \mathrm{~g}, 10 \mathrm{mmol})$ in dry pyridine $5 \mathrm{~mL}$ and dry DMF $2 \mathrm{~mL}$, at $-5^{\circ} \mathrm{C}$ a solution of terephthaloyl chloride (4.06 g, $20 \mathrm{mmol}$ ) in dry DMF 7 
$\mathrm{mL}$ was added dropwise under $\mathrm{N}_{2}$ atmosphere. The stirring was continued for half an hour at $-5^{\circ} \mathrm{C}$ and then overnight at room temperature. At the end of the reaction, for quench growth polymer solution was slowly trickled in to stirred methanol, giving rise to a stringy precipitate, which was washed thoroughly with hot methanol, collectedly filtration and dried at $60^{\circ} \mathrm{C}$ for $12 \mathrm{hrs}$ under vacuum to leave $90 \%$ of solid polymer.

\subsection{Film Fabrication}

Six polymer composite films were prepared from PAPA and PVA with different GR percentage as shown in Table 1. Each film as in Table 1 dissolved in $20 \mathrm{~mL}$ DMF and mixed completely under constant stirring for one hour while the mixture was heated up to $50^{\circ} \mathrm{C}$, then the mixture was left to cool down to room temperature $24^{\circ} \mathrm{C}$ with stirring of the mixture was carried out to ensure a homogenous composition. The obtained PAPA/PVA composite were mixed ultrasonically for 15-25 minutes with desired GR percentage composition as in Table 1 . To cast the films were poured the above mixtures in a casting glass plate $5 \times 5 \mathrm{~cm}$ and let it dry at room temperature for 120 hours. At the expiry of this time, the films were ready which were peeled off the casting glass plate.

Table 1. The compositions of the prepared films

\begin{tabular}{ccccc}
\hline Film Number & $\begin{array}{c}\text { PMPD } \\
(\mathrm{g} / \mathrm{film})\end{array}$ & $\begin{array}{c}\text { PVA } \\
(\mathrm{g} / \text { film })\end{array}$ & $\begin{array}{c}\text { Graphene } \\
(\mathrm{g} \% / \text { film })\end{array}$ & $\tau \times 10^{6}$ \\
\hline 1 & 0.8 & 1.2 & 0.000 & ---- \\
2 & 0.8 & 1.2 & 0.835 & 39.70 \\
3 & 0.8 & 1.2 & 1.655 & ---- \\
4 & 0.8 & 1.2 & 2.500 & 15.90 \\
5 & 0.8 & 1.2 & 3.500 & --- \\
6 & 0.8 & 1.2 & 4.165 & 7.95 \\
\hline
\end{tabular}

\subsection{Dielectric Constant Measurements}

The above fabricated films were cut into $2 \times 1.5 \mathrm{~cm}$ pieces to fit a homemade silver electrode for characterization by measuring dielectric properties using Precision LCR meter. The dielectric parameter as a function of frequency is described by the complex permittivity

$$
\varepsilon^{*}(\omega)=\varepsilon^{\prime}(\omega)-\varepsilon^{\prime \prime}(\omega)
$$

where the real part $\varepsilon^{\prime}$ and imaginary part $\varepsilon^{\prime \prime}$ are the components for the energy storage and energy loss, respectively, in each cycle of the electric field. $(\omega)$ is the angular frequency; $\omega=2 \pi f, f$ is applied frequency. The measured capacitance, $\mathrm{C}$ was used to calculate the dielectric constant, $\varepsilon^{\prime}$ using the following expression.

$$
\varepsilon^{\prime}=\frac{C d}{\varepsilon_{o} A}
$$

where $d$ is the thickness between the two electrodes (film thickness), $A$ is the area of the electrodes, $\varepsilon_{o}$ is permittivity of the free space, $\varepsilon_{o}=8.85 \times 10^{-12}$ F.m ${ }^{-1}$ and whereas for dielectric loss, $\varepsilon^{\prime \prime}(\omega)$ and $\tan \delta$ is tangent delta[22]:

$$
\varepsilon^{\prime \prime}(\omega)=\varepsilon^{\prime}(\omega) \cdot \tan \delta(\omega)
$$

The electric modulus is the reciprocal of the permittivity in complex form[23] was found using Eq. (4):

$$
M^{*}=1 /\left(\varepsilon^{*}\right)=M^{\prime}+M^{\prime \prime}
$$

where $M^{\prime}$ and $M^{\prime \prime}$ are the real and imaginary part of electric modulus and it was calculated by Eqs. (5 and 6 ):

$$
\begin{aligned}
M^{\prime} & =\varepsilon^{\prime} /\left(\varepsilon^{\prime 2}+\varepsilon^{\prime 2}\right) \\
M^{\prime \prime} & =\varepsilon^{\prime \prime} /\left(\varepsilon^{\prime 2}+\varepsilon^{\prime 2}\right)
\end{aligned}
$$

The relaxation times were obtained from renowned Havriliak Negami relationship Eq. 7[22].The angular frequency, $\omega^{\prime}$ can be obtained from the plotting of $M^{\prime \prime}$ versus $\log$ frequency.

$$
\begin{aligned}
\omega^{\prime} & =2 \pi f \\
\tau & =\frac{1}{\omega^{\prime}}
\end{aligned}
$$

The AC conductivity $(\sigma$ ac) can be calculated by the following equation[22]:

$$
\sigma=\varepsilon_{o} \varepsilon^{\prime} \omega^{\prime} \tan z
$$

\section{Results and Discussion}

Figure 2 shows several characteristic peaks of FT-IR for GO corresponding to $\mathrm{O}-\mathrm{H}$ vibration at $3200-3400 \mathrm{~cm}^{-1}, \mathrm{C}=\mathrm{O}$ stretching at $1726 \mathrm{~cm}^{-1}, \mathrm{C}=\mathrm{C}$ skeletal vibration from unoxidized graphitic domains at $1627 \mathrm{~cm}^{-1}$, O-H deformation at $1396 \mathrm{~cm}^{-1}$, epoxy symmetrical ring deformation at $1154 \mathrm{~cm}^{-1}, \mathrm{C}-\mathrm{O}$ stretching mixed with $\mathrm{C}-\mathrm{OH}$ bending at $1032 \mathrm{~cm}^{-1}$. After reduction of GO as seen in Figure 3, the peaks corresponding to $\mathrm{C}=\mathrm{O}$ stretching at 1726 $\mathrm{cm}^{-1}$, disappeared while the peak at $1557 \mathrm{~cm}^{-1}(\mathrm{C}=\mathrm{C})$ was retained, the stretching vibrations at $1060 \mathrm{~cm}^{-1}$ on the GO and reduced GO spectra indicate the presence of $\mathrm{C}-\mathrm{C}$ skeletal vibrations of the graphite ring. This showed that reduction results in the formation of $\mathrm{sp}^{2}$ carbon structure. Thus, it can be concluded that GR sheets were produced via reduction of GO.

The structure of PAPA polymer was confirmed by using elemental analysis and FT-IR spectroscopy. The observed values of $\mathrm{C}$ and $\mathrm{H}$ percentages (data not shown) of the repeating units are in agreement with theoretical values. In the spectrum of PAPA polymer, presented in Figure 4, the intense bands at 3402 and $1717 \mathrm{~cm}^{-1}$ were attributed to stretching vibrations, of the $\mathrm{O}-\mathrm{H}$ and $\mathrm{C}=\mathrm{O}$ stretching bands coming from pentaerythritol and terephthaloyl chloride monomers respectively. These analyses of this compound are good evidence which corresponds to the structure of suggested polymer. 


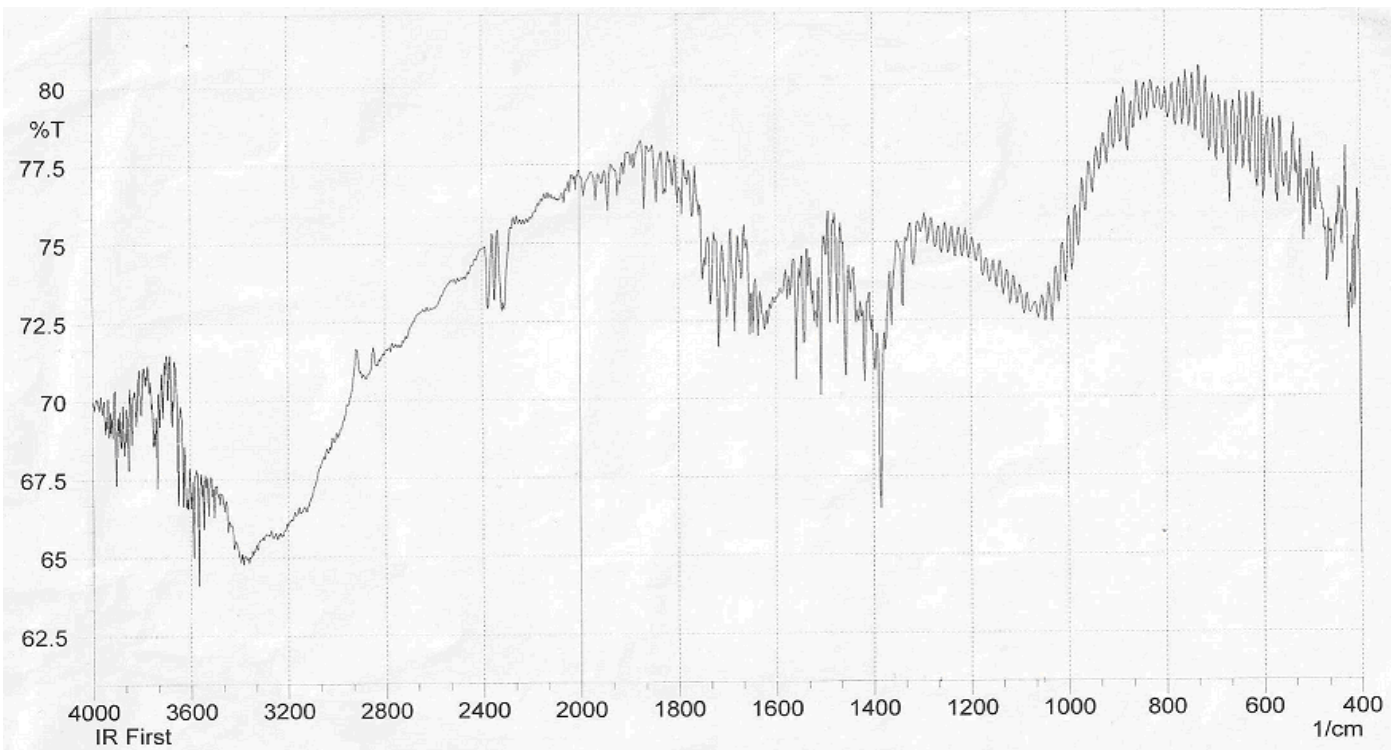

Figure 2. FT-IR spectra of GO

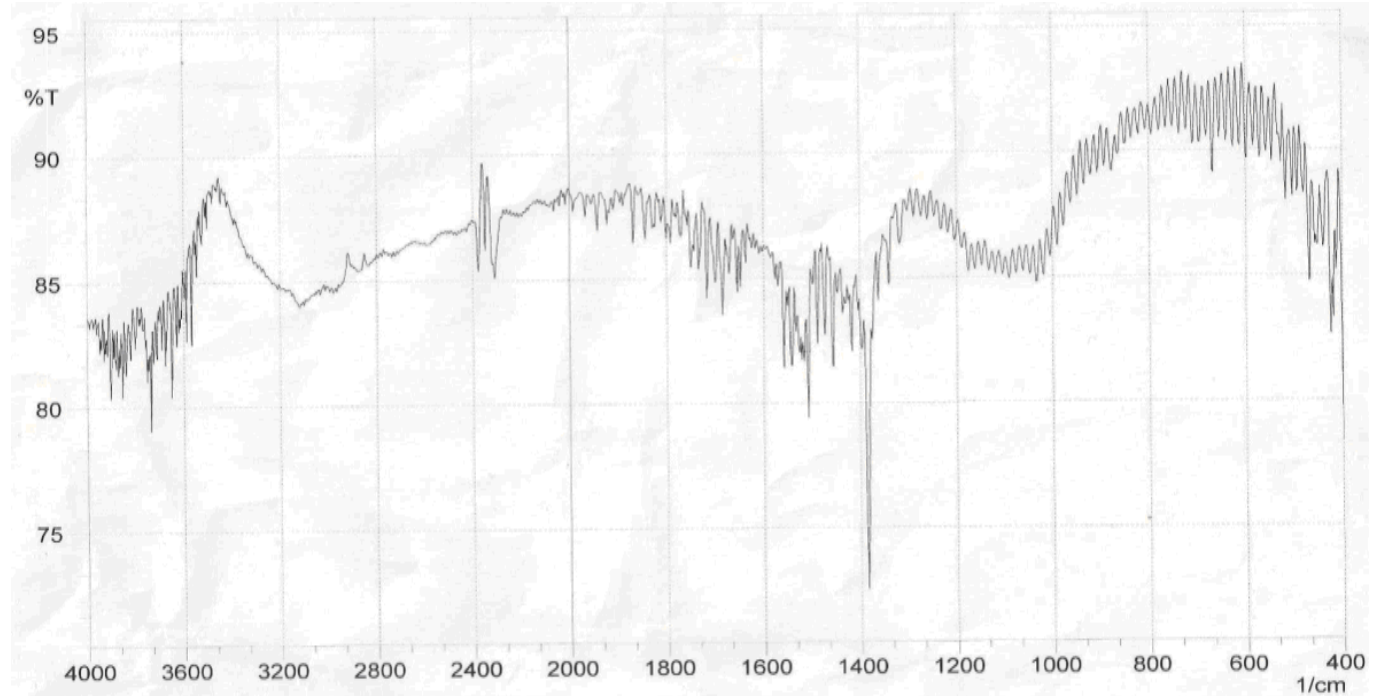

Figure 3. FT-IR spectra of GR sheets

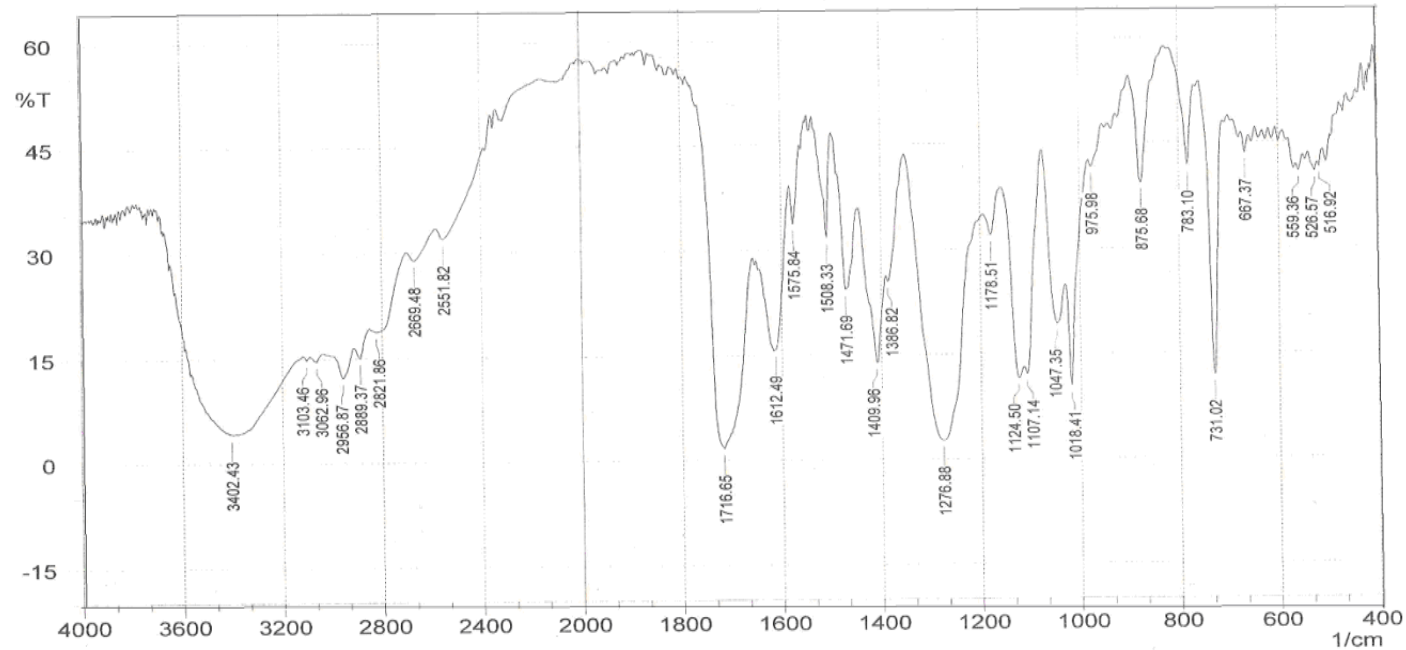

Figure 4. FT-IR spectrum of PAPA nanocomposite 
The XRD pattern of GO (Figure 5), the strong and sharp peak at $2 \theta=11.7^{\circ}$ corresponds to an interlayer distance of 7.6 $\AA$ (002). XRD pattern of reduced GO (Figure 6) shows a broad peak that can be fitted by using a Lorentzian function into three peaks cantered at $2 \theta=22.30^{\circ}, 25,68^{\circ}$ and $26.83^{\circ}$, corresponding to interlayer distances of 4.91, 3.72 and 3.39 $\AA$, respectively. These XRD results are related to the exfoliation and reduction processes of $\mathrm{GO}$ and the processes of removing intercalated water molecules and the oxide groups. All XRD patterns were identical with those obtained by Sungjin et. al., and Nethravathi et. al.,[24, 25].

The morphology of the prepared GR powder sample was examined by SEM and AFM, representative images are shown in Figures 7 and 8, respectively. The SEM reveals an agglomerated powder with a "fluffy" appearance. Figure 8 shows a high profile AFM image of GR. The suspensions concentration of GR was $(0.21 \mathrm{mg} / \mathrm{mL})$ and deposited on mica. It is known that the synthesis of GR materials from reduction of exfoliated GO generally yields samples which are not based on single separated GR sheets, but rather on an interconnected network with regions of over-lapped multiple layers[22, 23, 26]. This process also yields a highly agglomerated wrinkled sheet structure, resulting primarily from the high compliance of such extremely thin platelets, with a possible further contribution from reaction sites involved in oxidation and reduction processes[22, 23].

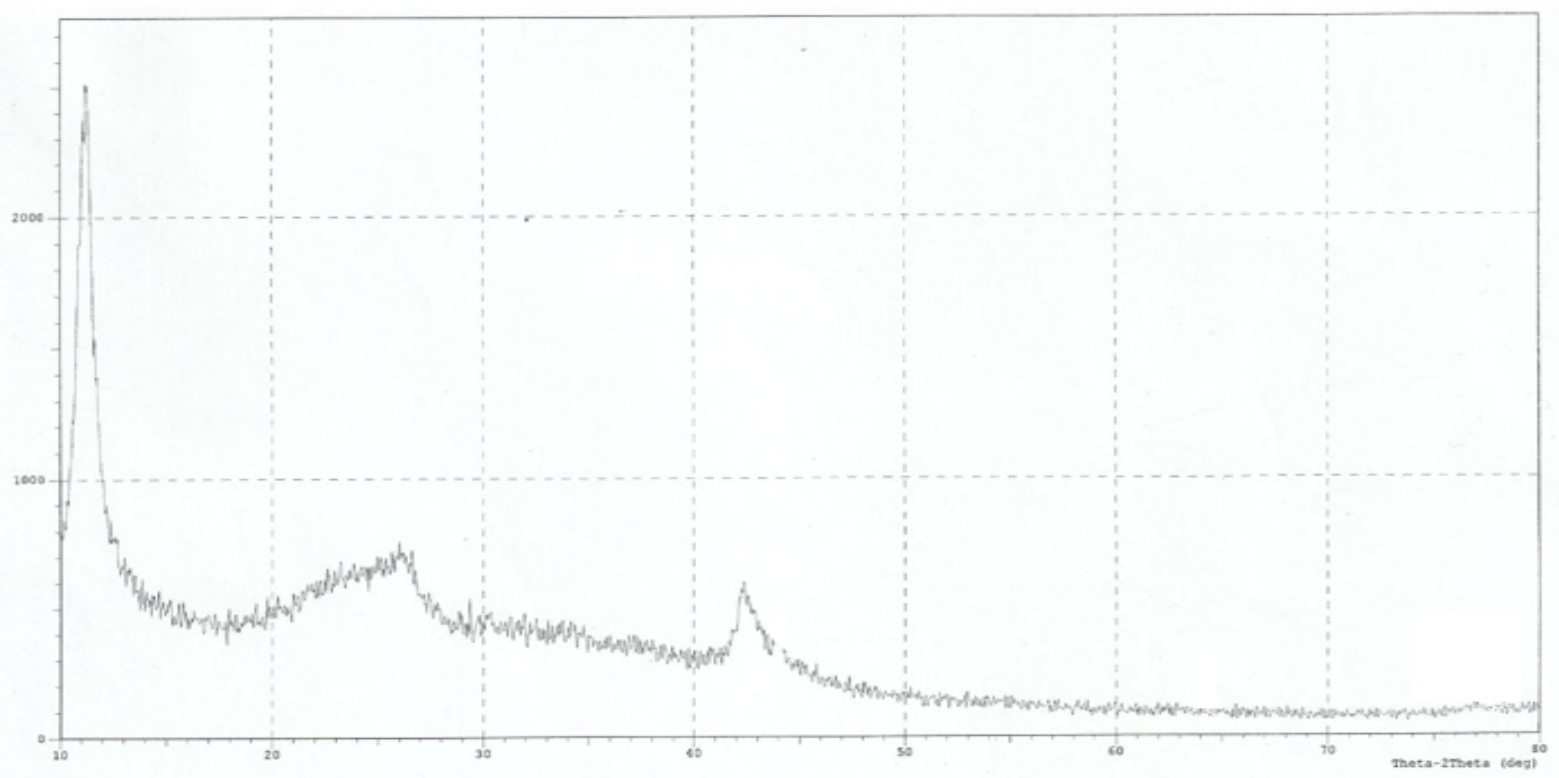

Figure 5. XRD patterns of GO

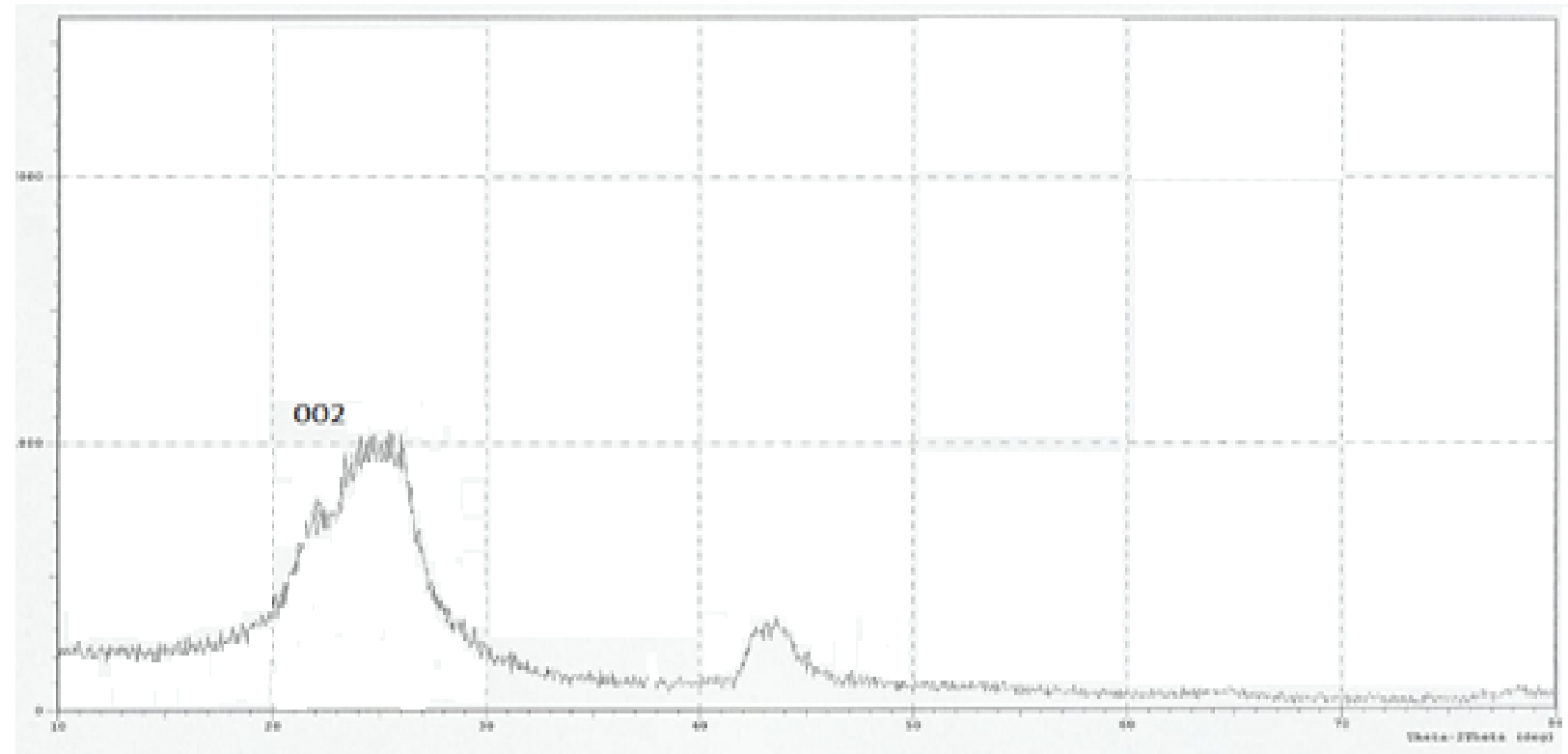

Figure 6. XRD pattern of GR powder sample 


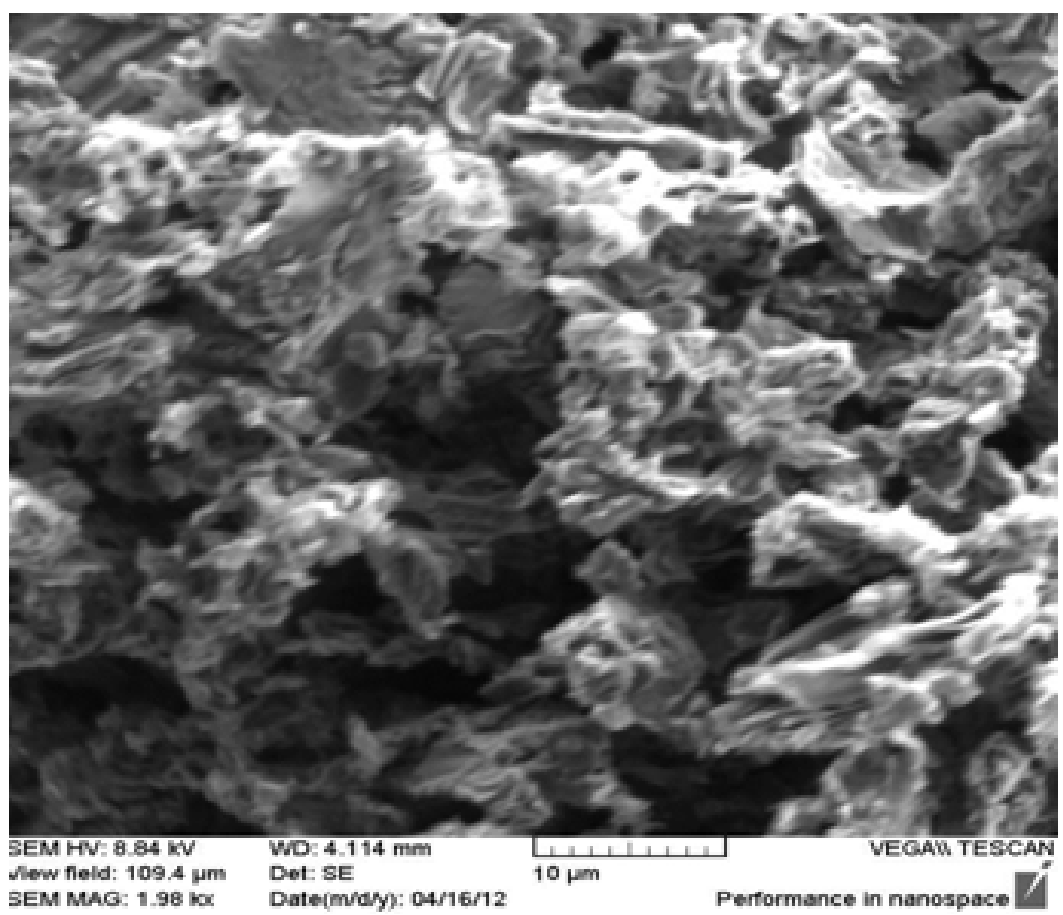

Figure 7. SEM image of GR sample

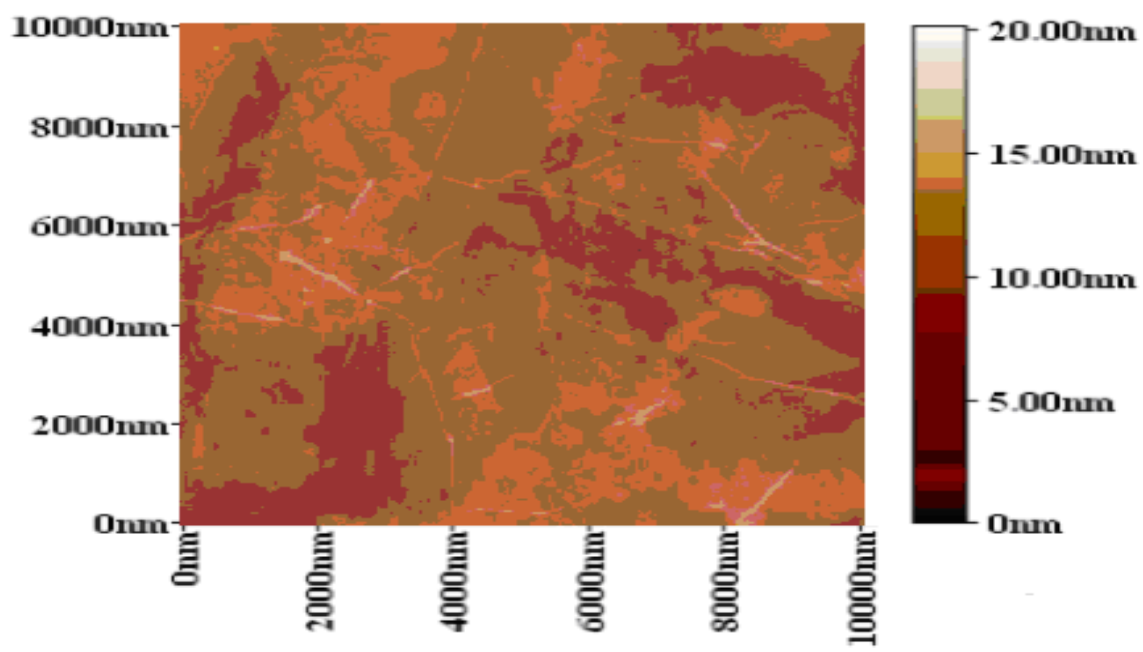

Figure 8. AFM image of GR sample

Evidence for stacks of GR sheets comes from the broad XRD out-of-plane graphitic reflections, Figs. 5 and 6. We label these peaks " $(002)$ ", as they occur at around the same position as that reflection from bulk graphite[23]. The number of stacked GR sheets and the in-plane crystallite size of the GR sample were estimated from the analysis of (002) and (100) XRD profiles. The (002) reflection peak is asymmetric and reveals two components behaviour. The inset of Figures 5 and 6 show a typical deconvoluted two phase multiple Lorentzian fit for the (002) reflection[27, 28]. These results were agreed with the results of Tantis et. al.,[15].

The interest in graphene-based composites stems from their potential high electrical conductivity and permittivity.
Dielectric data can be analysed by means of different formalisms, such as AC conductivity, dielectric permittivity, and electric modulus. Although, all three formalisms can be employed on the description and analysis of the occurring electrical effects. Our experimental data were firstly expressed via the AC conductivity, real and imaginary part of dielectric permittivity and then transformed into electric modulus formalism[29-31].

Figure 9 displays the frequency dependence of electrical properties. The variation of $\sigma$ (AC conductivity) was studied with frequency for different GR weight percentage content of composites at room temperature. At low GR weight percentage contents $(\leq 2.5 \%)$ were the electrical conductivity of the GR composites increases with increasing frequency. 
These specimens show a typical insulating behaviour with a frequency-dependent conductivity. When the GR percentage content reaches $3.5 \%$, there is a transition from an insulator to semiconductor. The transition filler concentration of the composite from insulating to conducting is defined as percolation threshold associated with the formation of conducting network[32]. So it can be seen that the change in the electrical conductivity depends on the amount of GR in the composites. At small amount, the conductivity of the GR composites increases with increasing frequency, i.e. when the GR distributed in the polymer matrix to form nanocomposites, it creates a lot of interfaces a large dominate of nomadic electron could provide with large $\pi$-orbital of the GR. The interface polarization can take place when electrons oriented under electric field[33]. Further increase of GR concentration will increase the number of interfaces but increase above a certain value will lead to the contact between the GR leading to the decrease of interfaces (percolation threshold), which will resulting in the decrease of the dielectric constant (Figure 9).

Figure 10 depicts the variation of real part of dielectric permittivity ( $\varepsilon^{\prime}$ ) with frequency for all GR composite percentage (Table 1 ) at constant temperature $\left(24^{\circ} \mathrm{C}\right)$. At low frequencies, permittivity attained higher values, in all cases, which diminished rapidly with frequency increasing. This is reasonable since in the low frequency region the alternation of the field is slow, providing thus sufficient time to permanent and induced dipoles to align themselves according to the applied field, leading to enhanced polarization. Enhanced values of ( $\varepsilon^{\prime}$ ) especially at low frequencies can be attributed interfacial polarization, and/or electrode polarization. Electrode polarization is related to the buildup of space charges at the specimen-electrode interfaces and is characterized by very high values of both real and imaginary part of dielectric permittivity[29-31]. Examined samples were tested under identical experimental conditions, having similar geometrical characteristics and composition. Thus, if electrode polarization was the prevalent effect in a certain type of nanocomposite, should be also the prevalent tendency to all other types. Under this point of view, the higher values of ( $\varepsilon^{\prime}$ ) could be attributed to enhanced conductivity and interfacial polarization (IP). IP results from the accumulation of unbounded charges at the interfaces of the constituents, where they form large dipoles; its intensity is connected to the extent of the existing interfacial area within the composite system, giving thus indirect evidence of the achieved distribution of nano inclusions[34]. Figure 10 was recorded a systematic increase of ( $\left.\varepsilon^{\prime}\right)$ with filler content for all samples. Since polarization and stored energy is directly proportional to permittivity. Dielectric loss, (Figure 11) was increased with increasing of GR composite percentage and the peaks shift to increase with decrease of the frequency.

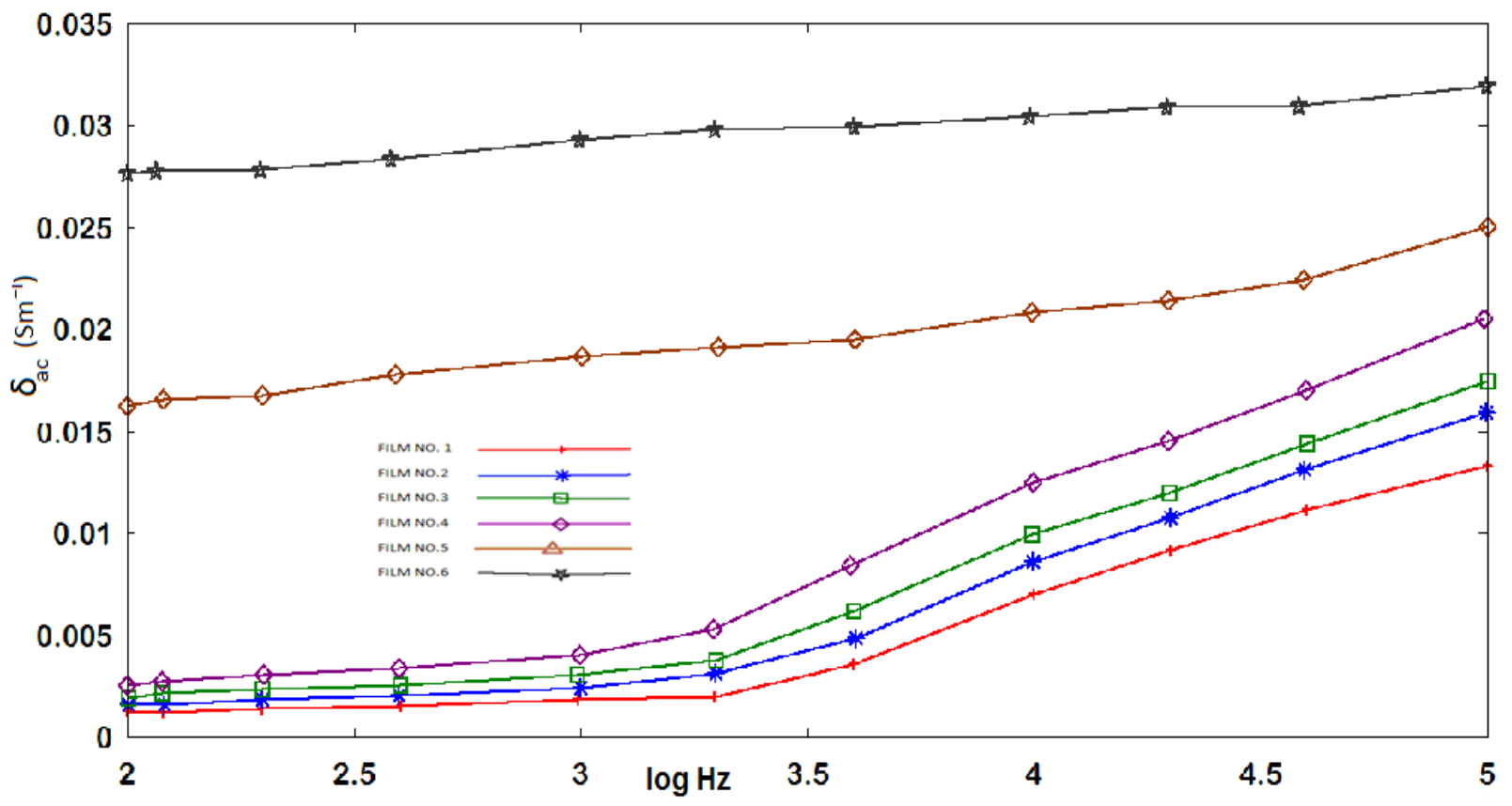

Figure 9. AC conductivity versus log frequency 


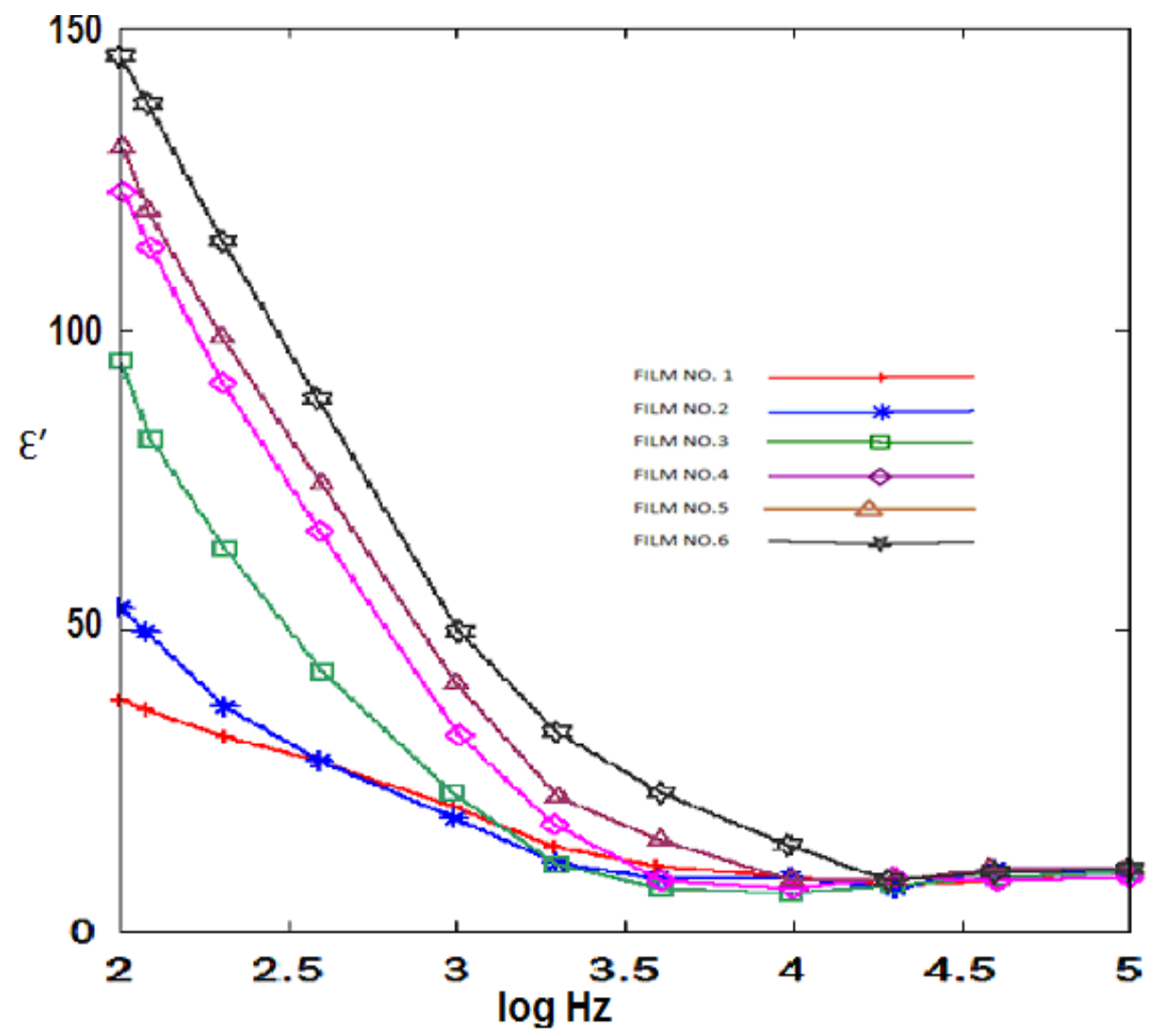

Figure 10. Real permittivity versus log frequency

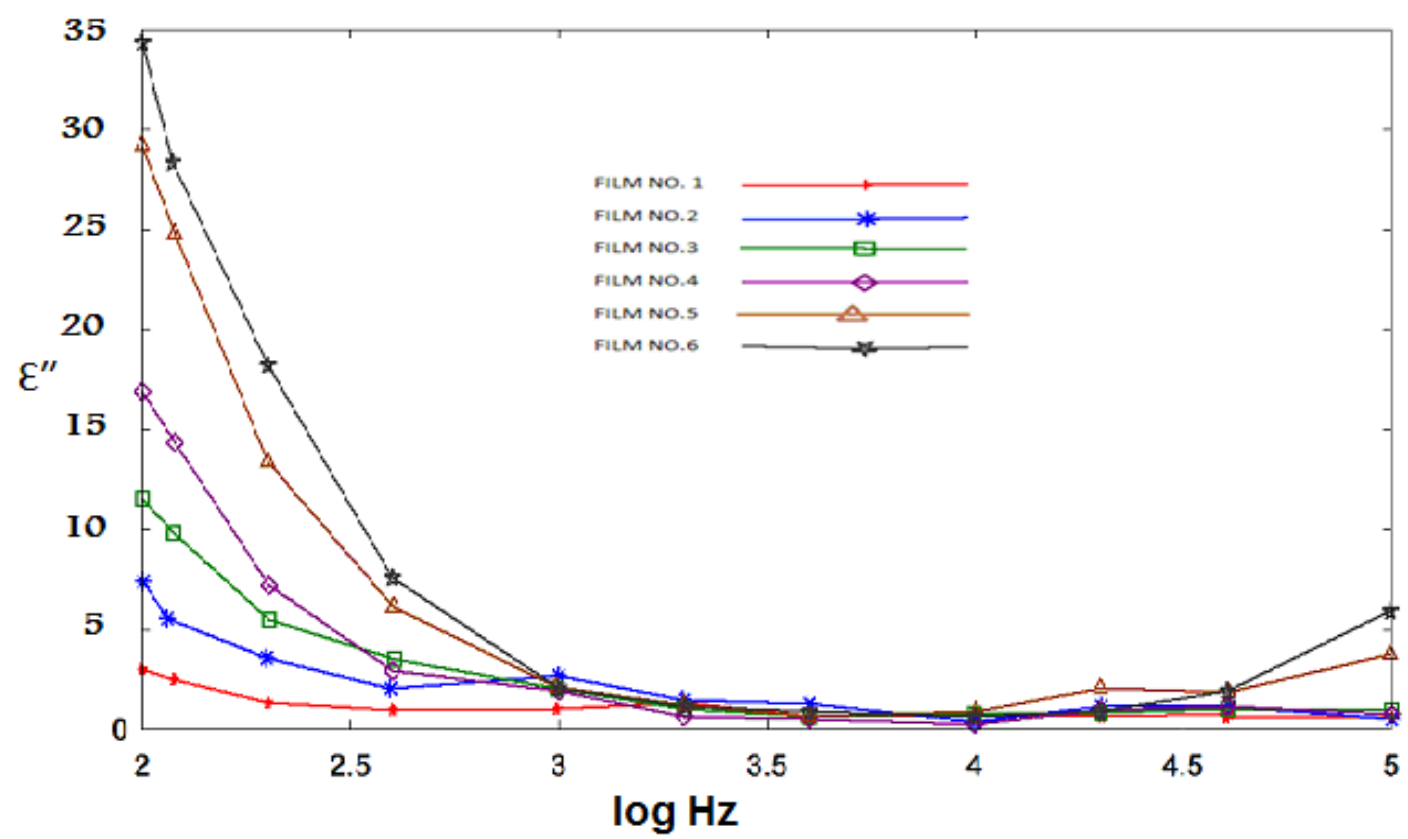

Figure 11. Imaginary permittivity versus $\log$ frequency

The variation of real and imaginary part of electric modulus ( $M^{\prime}, M^{\prime \prime}$ respectively) versus frequency for the PAPA/PVA/GR nanocomposite is depicted in Figures 12 and 13 respectively. The imaginary part of electric modulus $\left(M^{\prime \prime}\right)$ versus frequency spectra revealed to relaxation mechanism, which recorded at high frequencies, was attributed to interfacial polarization (IP). In Figure 13 the peak shifts to higher frequency with the increasing of GR composite concentrations. Figure 13 shows also that the height of the peak increase with the GR composite concentration as relaxation time $\tau$ decreases. The frequency at the maximum of the peak (Fig. 13) of $M^{\prime \prime}(\omega)$ is the relaxation frequency $\omega^{\prime}$ as tabulated in the inset in Figure 13. 


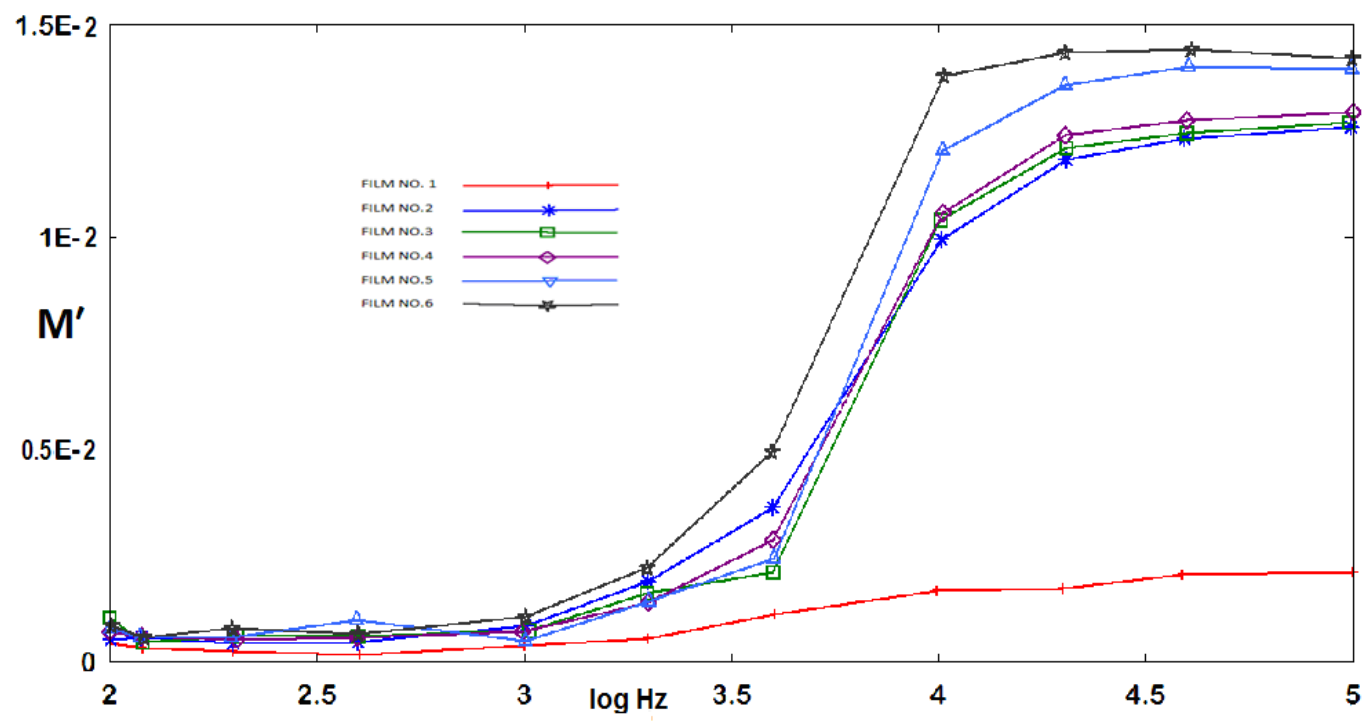

Figure 12. Real part of electrical modulus versus log frequency

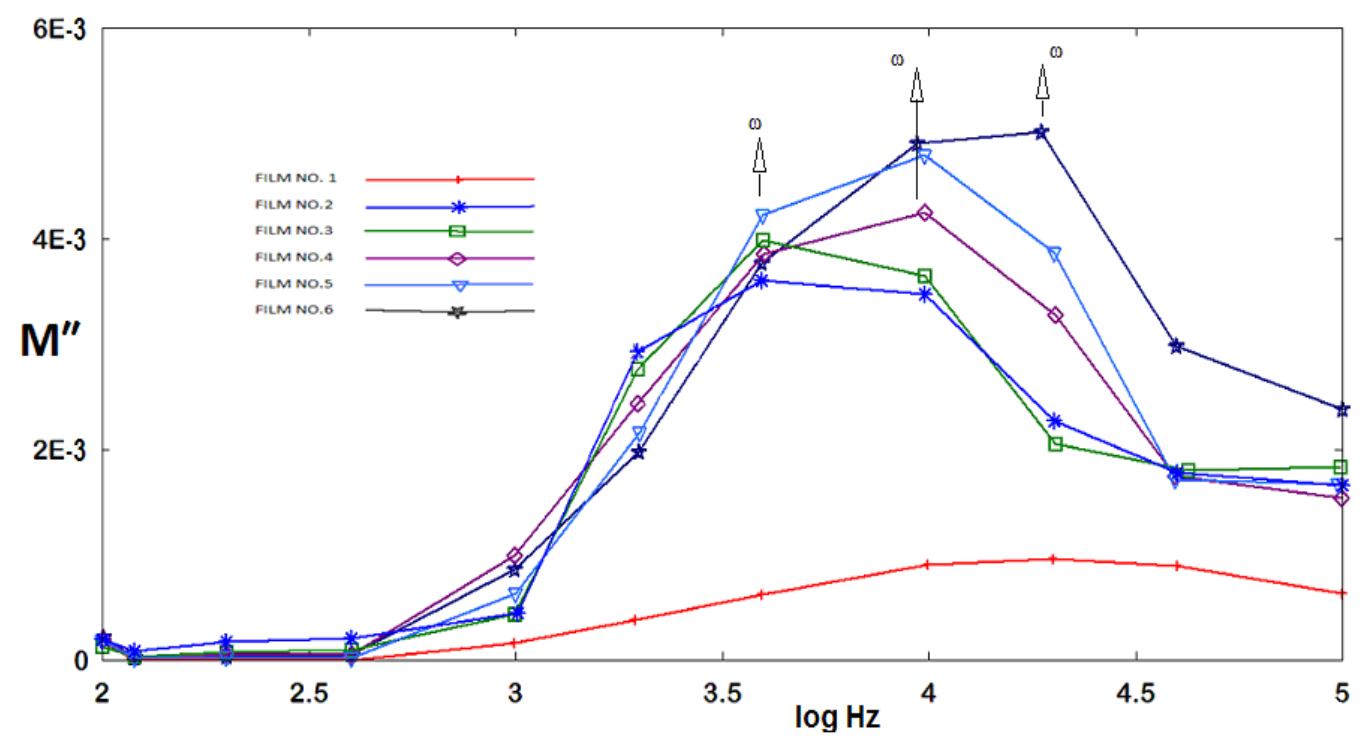

Figure 13. Imaginary part of electrical modulus versus log frequency

\section{Conclusions}

PAPA/PVA/GR composites were fabricated in six different GR composite percentages. The structural, morphology and electrical properties of the composites are characterized by x-ray diffraction (XRD), Fourier transform IR spectra (FT-IR), AFM and SEM. The electrical conductivity, dielectric properties and electric modulus of $\mathrm{PABA} / \mathrm{PVA} / \mathrm{GR}$ composites were measured in a range of frequencies from $100 \mathrm{~Hz}$ to $100 \mathrm{kHz}$. The results showed that the $\mathrm{AC}$ conductivity $(\sigma)$ and dielectric constant $\left(\varepsilon^{\prime}\right)$ of the $\mathrm{PABA} / \mathrm{PVA} / \mathrm{GR}$ composites were frequency dependent. At low GR weight percentage contents $(\leq 2.50 \%)$ the electrical conductivity of the GR composites increases with increasing frequency. These specimens show a typical insulating behaviour with a frequency-dependent conductivity, but when the GR weight percentage reach $4.165 \%$ the curve was showed a week slope which indicate that the DC conductivity increase continuously with increasing of GR weight percentages in the composite and the effect of the frequency became weaker on the conductivity and became more and more independent on the frequency. The behaviour of imaginary part of electrical modulus versus log frequency shows also that the height of the peak increase with the GR composite percentages as relaxation time decreases.

\section{REFERENCES}

[1] Geim AK "Graphene: Status and Prospects", Bentham Science Publisher, Science, vol. 324, no. 5934, pp.1530-1534, 2009.

[2] Geim AK, Novoselov K "The Rise of Graphene", Scientific Reports, Natural Materials, vol. 6, pp.183-191, 2007.

[3] Hancock Y, "The 2010 Nobel Prize in physics-groundbreaking experiments on graphen", IOPscience, Journal of 
Physics D: Applied Physics, vol. 44, no. 47, pp. 473001-4370013, 2011.

[4] Stankovich S, Dikin DA, Dommett GHB, Kohlhaas KM, Zimney EJ, Stach EA, Piner RD, Nguyen ST "Graphene-based composite materials", Nature Publishing Group, Nature, vol. 442, no. 7100, pp.282-286, 2006.

[5] Liu N, Luo F, Wu H, Liu Y, Zhang C, Chen J, "Onestep ionic-liquid-assisted electrochemical synthesis of ionicliquid- functionalized graphene sheets directly from graphite" Wiley, Advanced Functional Materials, vol. 18, no. 10, pp.1518-1525, 2008.

[6] Xu Y, Wang Y, Liang J, Huang Y, Ma Y, Wan X "A Hybrid Material of Graphene and Poly(3,4-ethyldioxythiophene) with High Conductivity, Flexibility, and Transparency", Spriger, Nano Research, vol. 2, no. 4, pp.343-348, 2009.

[7] Liang J, Xu Y, Huang Y, Zhang L, Wang Y, Ma Y "Infrared-triggered actuators from graphene-based nanocomposites", American Chemical Society, Journal of Physical Chemistry C, vol. 113, no. 22, pp.9921-9927, 2009.

[8] Zhu Y, Murali S, Cai W, Li X, Suk JW, Potts JR, Ruff RS "Graphene and graphene oxide: synthesis, properties, and applications", (2010) Advanced Materials, vol. 22, no. 35, pp.3906-3924, 2010.

[9] Compton OC, Nguyen SBT "Graphene oxide, highly reduced graphene oxide, and graphene: versatile building blocks for carbon-based materials", Wiley, Small, vol. 6, no. 6, pp.711-723, 2010.

[10] Carter LW, Hendricks JG, Bolley DS "Elastomer Reinforced With A Modified Clay", National Lead Co, US Patent 2531396, 1950 .

[11] Usuki A, Kojima Y, Kawasumi M, Okada A, Fukushima Y, Kurauchi T, Kamigaito O "Synthesis of nylon 6-clay hybrid", Springer, Journal of Materials Research, vol. 8, no. 5, pp.1179-1184, 1993.

[12] Winey KI, Vaia RA "Polymer nanocomposites" The Materials Getway, MRS Bull, vol. 32, pp.314-322, 2007.

[13] Ramanathan T, Abdala AA, Stankovich S, Dikin DA, Herrera AM, Piner RD, Admson DH, Schniepp HC, Chen X, Ruoff RS "Functionalized graphene sheets for polymer nanocomposites", Nature Nanotechnology, vol. 3, no. 6, pp.327-331, 2008.

[14] Dreyer DR, Park S, Bielawski CW, Ruoff RS "The chemistry of graphene oxide" RSC Publishing, Chemical Society Reviewes, vol. 39, pp.228-, 2010.

[15] Tantis, GC, Psarras D Tasis "Functionalized graphene poly(vinyl alcohol) nanocomposites: Physical and dielectric properties", SCI Mago lab, eXPRESS Polymer Letters, vol. 6, no. 4, pp.283-292, 2012.

[16] Kim H, Abdala AA, Macosko CW 'Graphene/polymer nanocomposites", American Chemical Society, Macromolecules, vol. 43, pp.6515-6530, 2010.

[17] Romasanta LJ, Hernández M, Manchado MAL, Verdejo R "Functionalised graphene sheets as effective high dielectric constant fillers", Springer, Nanoscale research letters , vol. 6 , pp.508-, 2011

[18] McAllister MJ, Li JL, Adamson DH, Schniepp HC, Abdala AA, Liu J, Herrera AM, Milius DL, Car R, Prud'homme RK,
Aksay IA "Single sheet functionalized graphene by oxidation and thermal expansion o graphite", American Chemical Society, Chemistry of Materials, vol. 19, pp.4396-4404, 2007.

[19] Verdejo R, Bernal MM, Romasanta JL, López-Manchado MA "Graphene filled polymer nanocomposites", RSC Publisher, Journal of Materials Chemistry, vol. 21, pp. 3301-3310, 2011.

[20] Hummers W, Offman R "Preparation of graphitic oxide" J. Am. Chem. Soc., vol. 80, pp.1339-, 1958.

[21] Zhenghai T, Chunfang Z, Yanda L, Baochun G, Liqun Z, Demin J "Fluorescent whitening agent stabilized graphene and its composites with chitosan" RSC Publisher, Journal of Materials Chemistry, vol. 21, no.43, pp.17111-17118, 2011.

[22] Stankovich S, Dikin DA, Piner RD, Kohlhaas KA, Kleinhammes A, Jia Y, Wu Y, Nguyen ST, Ruoff RS "Synthesis of graphene-basenanosheets via chemical reduction of exfoliated graphite oxide", Elsevier, Carbon, vol. 45, pp.1558-1565, 2007.

[23] Subrahmanyam KS, Vivekchand SRC, Govindaraj A, Rao A "A study of graphenes prepared by different methods: characterization, properties and solubilisation", RSC Publisher, Journal of Materials Chemistry, vol. 18, no.43, pp.1517-1523, 2008.

[24] Sungjin P, Jinho A, Potts JR, Velamakanni A, Murali S, Ruoff RS "Hydrazine-reduction of graphite and graphene oxide "Elsevier, Carbon, vol. 49, pp.3019-3023, 2011.

[25] Nethravathi C, Rajamathi M, Ravishankar N, Basit L, Felser $\mathrm{C}$ "Synthesis of graphene oxide-intercalated a-hydroxides by metathesis and their decomposition to graphene/metal oxide composites", Elsevier, Carbon, vol. 48, no.15, pp.4343-4350, 2010 .

[26] Ma LP, Wu ZS, Li J, Wu ED, Ren WC, Cheng HM "Hydrogen adsorption behavior of graphene above critical temperature", Elsevier, International Journal of Hydrogen Energy, vol. 34, pp.2329-2332, 2009.

[27] Park S, An J, Jung I, Piner RD, An SJ, Li X "Colloidal suspensions of highly reduced graphene oxide in a wide variety of organic solvents", Nano Letters, vol. 9, pp. 1593-1597, 2009.

[28] Li ZQ, Lu CJ, Xia ZP, Zhou Y, Luo Z "X-ray diffraction patterns of graphite and turbostratic carbon", Elsevier, Carbon, vol. 45, no.15, pp.1686-1695, 2007.

[29] Tsangaris GM, Psarras GC, Kouloumbi N "Polyurethane latex/water dispersible boehmite alumina nanocomposites: Thermal, mechanical and dielectrical properties", RSC Publisher, Journal of Materials Science,, vol. 33, no.8, pp.2027-2037, 1998.

[30] Gatos KG, Martínez AJG, Psarras GC, Thomann R Karger-Kocsis J Elsevier, Composites Science and Technology, vol. 67, no.2, pp.157-167, 2007.

[31] Psarras GC, Gatos KG, Karahaliou PK, Georga SN, Krontiras CA, Karger-Kocsis $\mathrm{J}$ "Relaxation phenomena in rubber/ layered silicate nanocomposites", Springer, Express Polymer Letters, vol. 1, pp.837-845, 2007.

[32] Li YC, Li RKY, Tjong SC "Frequency and temperature dependences of dielectric dispersion and electrical properties 
of polyvinylidene fluoride/expanded graphite composites "javascript:; Springer, Journal of Nanomaterials, no vol., and pp. given, 2010.

[33] Dang Z, Wang L, Yin Y, Zhang Q, Lei Q "Giant dielectric permittivities in functionalized carbonnanotube/ electroactive - polymer nanocomposites", Wiley, Advanced Materials, vol. 19, pp.852-857, 2007.
[34] Kalini A, Gatos KG, Karahaliou PK, Georga S N, Krontiras CA, Psarras GC "Probing the dielectric response of polyurethane/alumina nanocomposites", Journal of Polymer Science Part B: Polymer Physics, vol. 48, pp.2346-2354, 2010 . 